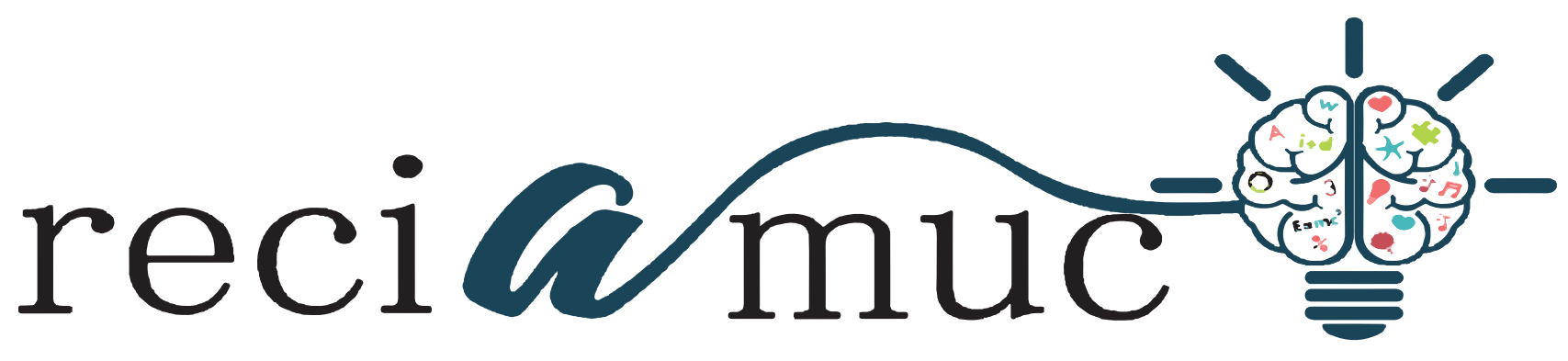

DOI: $10.26820 /$ reciamuc/4.(1).enero.2020.4-14

URL: https://reciamuc.com/index.php/RECIAMUC/article/view/423

EDITORIAL: Saberes del Conocimiento

REVISTA: RECIAMUC

ISSN: 2588-0748

TIPO DE INVESTIGACIÓN: Artículo de Revisión

CÓDIGO UNESCO: 3205 Medicina Interna

PAGINAS: $4-14$

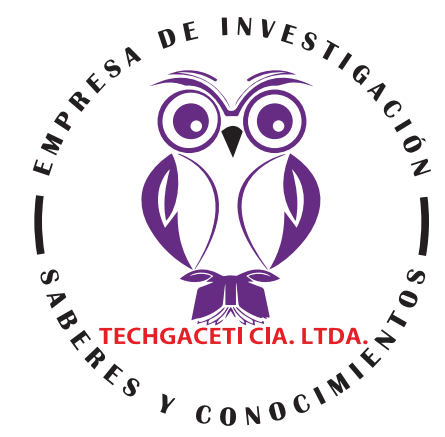

Necrosis avascular de la cabeza femoral

\title{
Avascular necrosis of the femoral head
} Necrose avascular da cabeça femoral

Cristhian Alexander Quinaluisa Erazo; Javier Alexander Veintimilla Pesantez²; María Ángela Pérez Vásquez;

Kevin David Aldás Ibujes ${ }^{4}$

RECIBIDO: 18/11/2019 ACEPTADO: 20/12/2019 PUBLICADO: 31/01/2020

1. Médico; Investigadora Independiente; Guayaquil, Ecuador; alex_trmf@hotmail.com; (D) https://orcid.org/00000003-1897-2441

2. Médico; Investigadora Independiente; Guayaquil, Ecuador; javier.Veintimilla@outlook.com; (DD "https://orcid. org/0000-0001-9379-2104

3. Médica Cirujana; Investigadora Independiente; Guayaquil, Ecuador; med92vpam@gmail.com; (D) "https:// orcid.org/0000-0002-8574-189X

4. Médico; Investigadora Independiente; Guayaquil, Ecuador; kdavid_1394@hotmail.com; (D) "https://orcid. org/0000-0002-2553-0548

CORRESPONDENCIA

Cristhian Alexander Quinaluisa Erazo

alex_trmf@hotmail.com

Guayaquil, Ecuador 


\title{
RESUMEN
}

La necrosis avascular (AVN) es una enfermedad que puede afectar varios huesos diferentes como resultado de la pérdida temporal o permanente del suministro de sangre a estos huesos. La cabeza femoral se ve afectada más comúnmente por esta enfermedad. Por lo general, los pacientes se encuentran en su tercera, cuarta o quinta década de vida al momento del diagnóstico. Inicialmente, los pacientes son asintomáticos, pero, con el tiempo, AVN conduce a la destrucción de las articulaciones, lo que requiere tratamiento quirúrgico y, en las últimas etapas, el reemplazo total de cadera. Es esencial que el AVN de la cabeza femoral se diagnostique temprano porque retrasar esta enfermedad mediante medidas de preservación articular tiene un pronóstico mucho mejor y porque los resultados del reemplazo articular son más pobres en individuos jóvenes. Hay varias condiciones que pueden incriminarse como desencadenantes de esta enfermedad. Sin embargo, casi la mitad de los pacientes diagnosticados no presentan ninguna de estas condiciones. Este tipo de necrosis avascular se denomina primaria o idiopática. Todas las otras formas de esta enfermedad son secundarias. El tratamiento se ha facilitado mediante el uso de sistemas de clasificación internacional ampliamente aceptados y mediante un diagnóstico precoz eficaz mediante MRI y otras técnicas de imagen. Aunque el tratamiento se ha vuelto cada vez más hacia la cirugía, no se ha desarrollado una terapia universalmente satisfactoria, incluso para la enfermedad temprana.

Palabras clave: Necrosis avascular, Cabeza femoral, Dolor de cadera, Clasificación Ficat-Arlet, Descompresión central, Reemplazo total de cadera.

\begin{abstract}
Avascular necrosis (AVN) is a disease that may affect several different bones as a result of temporary or permanent loss of the blood supply to these bones. The femoral head is most commonly affected by this disease. Usually, the patients are in their third, fourth or fifth decade of life at the time of diagnose. Initially, patients are asymptomatic, but, in time, AVN leads to joint destruction, requiring surgical treatment and, in latter stages, total hip replacement. It is essential that AVN of the femoral head is diagnosed early because delaying this disease by joint preserving measures has a much better prognosis and because the results of joint replacement are poorer in young individuals. There are various conditions than can be incriminated as triggers for this disease. However, almost half of the patients diagnosed present none of these conditions. This type of avascular necrosis is termed primary, or idiopathic. All the other forms of this disease are secondary. Treatment has been facilitated by using widely accepted international classification systems and by effective earlier diagnosis using MRI and other imaging techniques. Although treatment has turned more and more towards surgery, no universally satisfactory therapy has been developed, even for early disease.
\end{abstract}

Keywords: Avascular necrosis, Femoral head, Hip pain, Ficat-Arlet Classification, Core decompression, Total hip replacement.

\section{RESUMO}

A necrose avascular (AVN) é uma doença que pode afetar vários ossos diferentes como resultado da perda temporária ou permanente do suprimento sanguíneo para esses ossos. A cabeça femoral é mais comumente afetada por esta doença. Geralmente, os pacientes estão na terceira, quarta ou quinta década de vida no momento do diagnóstico. Inicialmente, os pacientes são assintomáticos, mas, com o tempo, a AVN leva à destruição articular, exigindo tratamento cirúrgico e, em estágios finais, reposição total do quadril. É essencial que o diagnóstico de AVN da cabeça femoral seja precoce, pois o atraso dessa doença por medidas de preservação articular tem um prognóstico muito melhor e porque os resultados da substituição articular são mais fracos em indivíduos jovens. Existem várias condições que podem ser incriminadas como gatilhos para esta doença. No entanto, quase metade dos pacientes diagnosticados não apresenta nenhuma dessas condições. Este tipo de necrose avascular é denominado primário ou idiopático. Todas as outras formas desta doença são secundárias. O tratamento foi facilitado pelo uso de sistemas de classificação internacional amplamente aceitos e pelo diagnóstico precoce eficaz usando ressonância magnética e outras técnicas de imagem. Embora o tratamento tenha se voltado cada vez mais para a cirurgia, nenhuma terapia universalmente satisfatória foi desenvolvida, mesmo para doenças precoces.

Palavras-chave: Necrose avascular, Cabeça femoral, Dor no quadril, Classificação Ficat-Arlet, Descompressão do núcleo, Artroplastia total do quadril. 


\section{Introducción}

La necrosis avascular (AVN), también conocida como osteonecrosis, necrosis aséptica o necrosis ósea isquémica, es una enfermedad que puede afectar varios huesos diferentes como resultado de la pérdida temporal o permanente del suministro de sangre a estos huesos. La isquemia causa la muerte y eventual colapso del tejido óseo, con su superficie articular suprayacente (Schoenstadt, 2008). Koenig fue el primero en describir esta condición, que denominó osteocondritis disecante, en 1888. En 1925, Haenish describió el primer caso que involucraba La cabeza femoral. En 1940, la causa de la necrosis se consideró la oclusión arterial. Pietrograndi describió el primer caso de AVN de la cabeza femoral después del uso de esteroides en 1957 (Aiello, 2008). Desde entonces, los científicos han descubierto cada vez más sobre la compleja fisiopatología de esta enfermedad, que hasta el día de hoy sigue siendo en gran medida desconocida. La cabeza femoral se ve afectada más comúnmente por esta enfermedad. Por lo general, los pacientes se encuentran en su tercera, cuarta o quinta década de vida al momento del diagnóstico. Los hombres son más propensos a esta enfermedad que las mujeres, la proporción de sexos es de aproximadamente.

Inicialmente, los pacientes son asintomáticos, pero, con el tiempo, AVN conduce a la destrucción de las articulaciones, lo que requiere tratamiento quirúrgico y, en las últimas etapas, reemplazo total de cadera (THR). Aunque el tratamiento se ha facilitado mediante el uso de un sistema de clasificación internacional ampliamente aceptado, un diagnóstico previo efectivo mediante resonancia magnética y un tratamiento quirúrgico más agresivo, no se ha desarrollado una terapia universalmente satisfactoria, incluso para la enfermedad temprana.

Es esencial que el AVN de la cabeza femoral se diagnostique temprano porque retrasar esta enfermedad mediante medidas de preservación articular tiene un pronóstico mucho mejor y porque los resultados del reemplazo articular son más pobres en individuos jóvenes (Aiello, 2008).

\section{Metodología}

Para el desarrollo de este proceso investigativo, se plantea como metodología la encaminada hacia una orientación científica particular que se encuentra determinada por la necesidad de indagar en forma precisa y coherente una situación, en tal sentido (Davila, 2015) define la metodología "como aquellos pasos previos que son seleccionados por el investigador para lograr resultados favorables que le ayuden a plantear nuevas ideas".(p.66)

Lo citado por el autor, lleva a entender que el desarrollo de la acción investigativa busca simplemente coordinar acciones enmarcadas en una revisión bibliográfica con el fin de complementar ideas previas relacionadas a la necrosis avascular a través de una revisión de literatura, para así finalmente elaborar un cuerpo de consideraciones generales que ayuden a ampliar el interés propuesto.

\section{Tipo de Investigación}

Dentro de toda práctica investigativa, se precisan acciones de carácter metodológico mediante las cuales, se logra conocer y proyectar los eventos posibles que la determinan, así como las características que hacen del acto científico un proceso interactivo ajustado a una realidad posible de ser interpretada. En este sentido, se puede decir, que la presente investigación corresponde al tipo documental, definido por Castro (2016), "se ocupa del estudio de problemas planteados a nivel teórico, la información requerida para abordarlos se encuentra básicamente en materiales impresos, audiovisuales y /o electrónicos". (p.41).

En consideración a esta definición, la orientación metodológica permitió la oportunidad de cumplir con una serie de actividades in- 
herentes a la revisión y lectura de diversos documentos donde se encontraron ideas explicitas relacionadas con los tópicos encargados de identificar a cada característica insertada en el estudio. Por lo tanto, se realizaron continuas interpretaciones con el claro propósito de revisar aquellas apreciaciones o investigaciones propuestas por diferentes investigadores relacionadas con el tema de interés, para luego dar la respectiva argumentación a los planteamientos, en función a las necesidades encontradas en la indagación.

\section{Fuentes Documentales}

El análisis correspondiente a las características que predomina en el tema seleccionado, llevan a incluir diferentes fuentes documentales encargadas de darle el respectivo apoyo y en ese sentido cumplir con la valoración de los hechos a fin de generar nuevos criterios que sirven de referencia a otros procesos investigativos. Para (CASTRO, 2016) las fuentes documentales incorporadas en la investigación documental o bibliográfica, "representa la suma de materiales sistemáticos que son revisados en forma rigurosa y profunda para llegar a un análisis del fenómeno".(p.41). Por lo tanto, se procedió a cumplir con la realización de una lectura previa determinada para encontrar aquellos aspectos estrechamente vinculados con el tema, con el fin de explicar mediante un desarrollo las respectivas apreciaciones generales de importancia.

\section{Técnicas para la Recolección de la Infor- mación}

La conducción de la investigación para ser realizada en función a las particularidades que determinan a los estudios documentales, tiene como fin el desarrollo de un conjunto de acciones encargadas de llevar a la selección de técnicas estrechamente vinculadas con las características del estudio. En tal sentido, (Bolívar, 2015), refiere, que es "una técnica particular para aportar ayuda a los procedimientos de selección de las ideas primarias y secundarias". (p. 71).
Por ello, se procedió a la utilización del subrayado, resúmenes, fichaje, como parte básica para la revisión y selección de los documentos que presentan el contenido teórico. Es decir, que mediante la aplicación de estas técnicas se pudo llegar a recoger informaciones en cuanto a la revisión bibliográfica de los diversos elementos encargados de orientar el proceso de investigación. Tal como lo expresa, (Bolívar, 2015) "las técnicas documentales proporcionan las herramientas esenciales y determinantes para responder a los objetivos formulados y llegar a resultados efectivos" (p. 58). Es decir, para responder con eficiencia a las necesidades investigativas, se introdujeron como técnica de recolección el método inductivo, que hizo posible llevar a cabo una valoración de los hechos de forma particular para llegar a la explicación desde una visión general.

Asimismo, se emplearon las técnicas de análisis de información para la realización de la investigación que fue ejecutada bajo la dinámica de aplicar diversos elementos encargados de determinar el camino a recorrer por el estudio, según, (Bolívar, 2015) las técnicas de procesamiento de datos en los estudios documentales "son las encargadas de ofrecer al investigador la visión o pasos que debe cumplir durante su ejercicio, cada una de ellas debe estar en correspondencia con el nivel a emplear" (p. 123). Esto indica, que para llevar a cabo el procesamiento de los datos obtenidos una vez aplicado las técnicas seleccionadas, tales como: fichas de resumen, textual, registros descriptivos entre otros, los mismos se deben ajustar al nivel que ha sido seleccionado.

\section{Resultados}

Hay varias condiciones que pueden estar en criminizadas como desencadenantes de esta enfermedad. Sin embargo, casi la mitad de los pacientes diagnosticados no presentan ninguna de estas condiciones. Este tipo de necrosis avascular se denomi- 
na primaria o idiopática. Todas las otras formas de esta enfermedad son secundarias (Marti-Carvajal, Dunlop, \& Agreda-Perez, 2004). Una de las razones más comunes para la necrosis avascular secundaria es el uso prolongado de esteroides sistémicos en dosis altas (equivalente a $4000 \mathrm{mg}$ de prednisona) durante períodos prolongados de hasta 3 meses o más. Hubo casos citados en la literatura de AVN de la cabeza femoral después de períodos relativamente breves (7 días) de esteroides orales. El mecanismo de acción aún no se comprende completamente, pero se cree que está relacionado con el estado hipercoagulable, con fibrinólisis posterior deteriorada y trombosis venosa en la cabeza femoral. Otras causas comunes incluyen trauma, trastornos sanguíneos o enfermedad de descompresión.

\section{Fisiopatología}

Los factores arteriales extraóseos son los más importantes. La cabeza femoral está en mayor riesgo porque el suministro de sangre es un sistema de órganos terminales con un pobre desarrollo colateral. El suministro de sangre puede ser interrumpido por trauma, vasculitis (enfermedad de Raynaud) o vasoespasmo (enfermedad de descompresión) (Aldridge \& Urbaniak, 2004).

Los factores arteriales intraóseos pueden bloquear la microcirculación de la cabeza femoral a través de micro embolias circulantes. Estos pueden ocurrir en la enfermedad de células falciformes (SCD), embolización de grasa o embolización de aire por fenómenos disbáricos.

Los factores venosos intraóseos afectan la cabeza femoral al reducir el flujo sanguíneo venoso y causar estasis. Estos factores pueden acompañar condiciones como la enfermedad de Caisson, SCD o el agrandamiento de las células grasas intramedulares (Marti-Carvajal, Dunlop, \& Agreda-Perez, Treatment for avascular necrosis of bone in people with sickle cell disease., 2004).

Los factores extravasculares intraóseos afectan la cadera al aumentar la presión, lo que resulta en un síndrome del compartimento de la cabeza femoral. Por ejemplo:

- La hipertrofia de las células grasas después de la administración de esteroides o las células anormales, como Gaucher y las células inflamatorias, pueden invadir los capilares intraóseos, reduciendo la circulación intramedular y contribuyendo al síndrome compartimental.

- Las microfracturas repetidas en el segmento de carga del fémur pueden causar múltiples lesiones vasculares que resultan en isquemia dentro del hueso frágil y mal reparado.

- Los factores citotóxicos, como el alcoholismo y el uso de esteroides, tienen un efecto metabólico tóxico directo sobre las células osteogénicas.

- La disminución de las concentraciones de 1,25 dihidroxivitamina D3 puede causar una deficiencia cuantitativa o cualitativa en la arquitectura ósea, haciendo que el hueso se deforme bajo presión.

Los factores extravasculares extravasculares (capsulares) implican el taponamiento de los vasos epifisarios laterales ubicados dentro de la membrana sinovial, a través del aumento de la presión intracapsular. Esto ocurre después de un traumatismo, infección y artritis, causando derrame que puede afectar el suministro de sangre a la epífisis (Aiello, 2008).

\section{Clinical details}

\section{History}

Avascular necrosis may be asymptomatic and is occasionally discovered following radiography.

Pain in the affected joint, described as throbbing, deep and, often, intermittent, is typically the presenting symptom. Patients with AVN of the femoral head often report groin or hip pain that can radiate to the buttoc$\mathrm{ks}$, anteromedial thigh, or knee that is exa- 
cerbated by weight bearing and sometimes by coughing. The pain may initially be mild but progressively worsens over time and with use. Eventually, the pain is present at rest and may be present or even worsen at night, in which case, it may be associated with morning stiffness.

\section{Físico}

1. Por lo general, los hallazgos iniciales no son reveladores.

2. En las últimas etapas de la enfermedad, la función articular se deteriora y se pueden encontrar los siguientes signos:

- El paciente puede caminar con cojera y puede experimentar pérdida de rango de movimiento, tanto activo como pasivo, con mayor frecuencia en flexión, abducción y rotación interna, especialmente después del colapso de la cabeza femoral.

- El paciente puede tener sensibilidad alrededor del área afectada.

- Se puede encontrar un déficit neurológico.

- El signo de Trendelenburg puede ser positivo.

- Se puede escuchar un clic cuando el paciente se levanta de una silla o después de la rotación externa de la cadera abducida.

3. La enfermedad avanzada conduce a la deformidad articular y al desgaste muscular (Jeanne, Tofferi, \& Gilliland, 2008).

\section{Imagen}

Los hallazgos radiográficos en película simple se presentan en las etapas 0 y 1 de AVN. Una radiografía normal no es igual a una cadera normal. Puede producirse un retraso de 1 a 5 años entre los primeros síntomas y la aparición de modificaciones radiográficas. En enfermedades más avanzadas, las radiografías muestran esclerosis y cambios en la densidad ósea. A medida que la enfermedad progresa, pueden aparecer líneas radiolúcidas subcondrales (signo creciente), aplanamiento o colapso de la cabeza femoral.

La tomografía computarizada se usa para determinar el grado de afectación, como las lucencias subcondrales y las esclerosis presentes en la etapa reparadora (antes del colapso de la cabeza femoral), pero no es tan sensible como la MRI en las etapas 0 y 1. La tomografía computarizada es excelente para detectar el colapso de la cabeza femoral, la enfermedad articular degenerativa temprana y la presencia de cuerpos sueltos, especialmente cuando se usa la reconstrucción multiplanar.

La resonancia magnética es el medio más sensible para diagnosticar AVN, representando el estándar de oro de la evaluación diagnóstica no invasiva (FIGURA 1). Tiene varias ventajas:

- Permite una estadificación precisa al representar claramente el tamaño de la lesión.

- Detecta lesiones asintomáticas que son indetectables en radiografías simples, lo que facilita el tratamiento temprano y una mejor respuesta.

- Proporciona imágenes multiplanares y excelente resolución de tejidos blandos.

- Puede demostrar la respuesta de la cabeza femoral al tratamiento.

La tomografía computarizada por emisión de fotón único (SPECT) se utiliza como alternativa para la resonancia magnética cuando esta última no se puede realizar o cuando los resultados de la resonancia magnética no están determinados. SPECT es difícil de usar porque requiere permanecer quieto durante largos períodos de tiempo. Además, los artefactos de la vejiga son un problema frecuente (Sarikaya, Sarikaya, \& Holder). 
Las imágenes escintigráficas revelan un área central de captación disminuida, rodeada por un área de captación incrementada (el signo del donut o el signo del frío en caliente) (Aiello, 2008). Indica la zona reactiva que rodea el área necrótica. Las principales desventajas son que carece de especificidad y que la misma imagen puede encontrarse en otras afecciones, como la osteoartritis, las fracturas y la artritis inflamatoria. Los resultados son difíciles de interpretar si la enfermedad es bilateral.
La biopsia ósea no se usa de forma rutinaria debido a la disponibilidad de pruebas sensibles no invasivas como la resonancia magnética (Jeanne, Tofferi, \& Gilliland, Avascular Necrosis, 2008). Es una valiosa herramienta de diagnóstico después de analizar el fragmento óseo extraído después de la descompresión del núcleo.

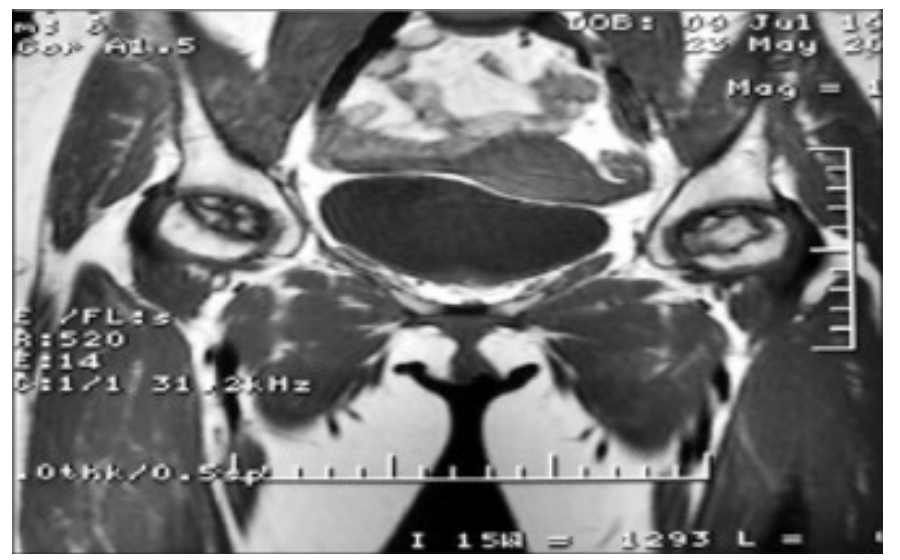

Figura 1. Imagen de resonancia magnética de lesión bilateral tipo II

Fuente: Autores 2020

\section{Diagnóstico diferencial}

- Trauma.

- Enfermedad degenerativa.

- Osteoporosis.

- Artritis.

- Sinovitis inflamatoria.

- Displasia epifisaria.

- Fractura de estrés epifisario.

- Osteoporosis transitoria de la cadera.

- Osteomielitis.

- Malignidad.

- Hemangioma.

- Terapia de radiación.
- Distrofia simpática.

- Síndrome de edema de médula ósea.

\section{Clasificación}

Ficat y Arlet han desarrollado un sistema de estadificación utilizando hallazgos radiográficos, que consta de cuatro etapas. Hungerford y Lennox modificaron este sistema de estadificación cuando MRI estuvo disponible, agregando la etapa 0 a la clasificación (Aldridge \& Urbaniak, 2004).

Etapa 0 (preclínica y preradiológica): hallazgos negativos en una radiografía simple, en un paciente asintomático con diagnóstico positivo en la cadera contralateral. La resonancia magnética muestra un signo de doble línea, consistente con un proceso necrótico. 
Etapa I (preradiológica): hallazgos normales en radiografías y hallazgos positivos en resonancia magnética o gammagrafía ósea. La etapa 1 representa la etapa de resorción temprana. Los primeros hallazgos radiográficos aparecen tarde en esta etapa de resorción (osteoporosis mínima y / o desenfoque y una definición deficiente de las trabéculas óseas).

La etapa II (etapa de reparación) ocurre antes del aplanamiento de la cabeza femoral. Puede durar varios meses o años. Los cambios radiográficos son evidentes y están representados por la desmineralización (manifestación temprana de la etapa reparativa, que representa la reabsorción del hueso muerto) y la esclerosis (que aparece después de la desmineralización, que representa la aposición de hueso nuevo en las trabéculas muertas). La desmineralización puede ser generalizada o irregular o aparecer en forma de pequeños quistes dentro de la cabeza femoral. La esclerosis irregular aparece como una mayor densidad en las radiografías, generalmente en el aspecto superolateral de la cabeza femoral y puede ser difusa, focal o en un arco lineal, que es cóncavo en la parte superior.

El estadio III (colapso temprano de la cabeza femoral) se anuncia por la presencia de secuestro y depresión, con la aparición de decrochaje, sin afectación acetabular. La cabeza femoral ya no es redonda y está suavemente torcida. Parece aplanado o colapsado. (Figura 3)

El estadio IV (enfermedad degenerativa progresiva) está representado por el colapso severo y la destrucción de la cabeza femoral junto con el estrechamiento del espacio articular, la formación de quistes osteofitos y subcondrales, como signos definitivos de enfermedad articular degenerativa.

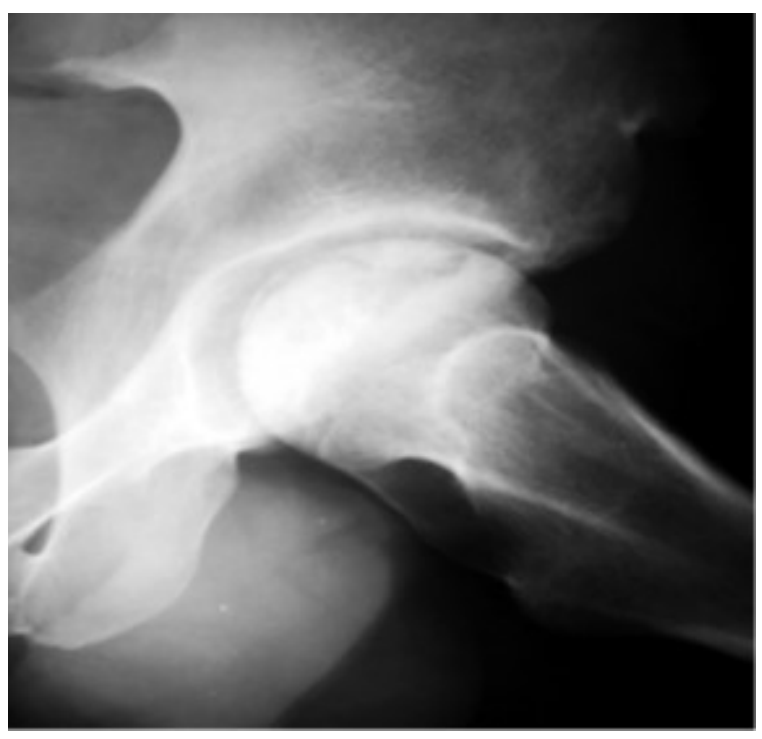

Figura 2. Signo de media luna

Fuente: Autores 2020

\section{Tratamiento}

El objetivo del tratamiento es evitar que las articulaciones se rompan, porque el dolor intenso y la limitación en el movimiento ocurrirán dentro de 2 años, en ausencia de tratamiento. Hay varias opciones entre las que se puede elegir y para determinar el trata- miento más apropiado, se debe tener en cuenta la edad del paciente, la etapa de la enfermedad, la ubicación y la cantidad de hueso afectado y la causa subyacente de la avascular. necrosis (se interrumpe el uso de corticosteroides o alcohol, el tratamiento puede no funcionar) (Schoenstadt, 2008). 
Existen métodos conservadores y quirúrgicos para tratar esta enfermedad. Los tratamientos conservadores se han utilizado experimentalmente solos o en combinación, pero rara vez proporcionan una mejora duradera. La mayoría de los pacientes eventualmente necesitarán cirugía, ya sea para retrasar o incluso para reparar la articulación de forma permanente (Schoenstadt, 2008).

\section{Tratamiento conservador}

- La terapia con estatinas, bifosfonatos o medicamentos antiinflamatorios no esteroideos pueden ser útiles.

- En algunos casos tempranos, la reducción de peso, las actividades limitantes o el uso de muletas pueden retrasar el daño causado por la necrosis avascular y permitir la curación natural. Sin embargo, estos pacientes corren un riesgo de 85\% de colapso de la cabeza femoral. El soporte de peso protegido puede ser efectivo cuando el segmento involucrado es menor al $15 \%$ y está ubicado lejos de la región de soporte de peso.

- Los ejercicios de rango de movimiento son útiles para mantener la función articular.

- Se cree que la estimulación eléctrica induce el crecimiento óseo.

\section{Tratamiento quirúrgico}

- La descompresión del núcleo se logra al mover la capa interna del hueso y proporciona un alivio inmediato del dolor al reducir la presión en el hueso, disminuir la congestión vascular y la inflamación y aliviar el síndrome compartimental. Mejora el proceso de sustitución progresiva y también estimula la formación de nuevos vasos sanguíneos, aumentando así el flujo sanguíneo al hueso. Está indicado en personas con etapas tempranas de necrosis avascular, antes del colapso de la cabeza y cuando está involucrado menos del $30 \%$ de la cabeza femo- ral (Aiello, 2008). La descompresión del núcleo también es efectiva para aliviar el dolor y ayuda a retrasar la necesidad de una artroplastia, actuando como un protector de las articulaciones.

- El injerto óseo utiliza hueso sano de una parte del paciente y lo trasplanta al área enferma. Después del fracaso de los injertos no vascularizados, los injertos actuales (llamados injertos vasculares) incluyen una arteria y venas, lo que aumenta el suministro de sangre al área afectada. El injerto óseo se puede combinar con la descompresión del núcleo, actuando juntos para detener el ciclo de isquemia. Está indicado en las primeras etapas de la enfermedad y, cuando tiene éxito, puede garantizar la supervivencia de la cabeza femoral de por vida, en ausencia de complicaciones asociadas a cuerpos extraños. Si no tiene éxito, el procedimiento permite al paciente retener la opción de artroplastia total de cadera en el futuro.

- La osteotomía es un procedimiento mediante el cual se reforma el hueso para reducir el estrés en el área afectada. Requiere un período de recuperación más largo y actividades limitadas durante 3 a 12 meses después de la cirugía. Este procedimiento es más efectivo para pacientes con necrosis avascular avanzada y aquellos con una gran área de hueso afectado. Las osteotomías rotacionales entre trocánteres y transtrocantéreos tienen como objetivo llevar un área intacta de hueso y cartílago al área de soporte de peso de la cabeza femoral y, al mismo tiempo, mejorar el suministro de sangre (efecto biotrófico). El consenso es que las osteotomías femorales se deben realizar en etapas más avanzadas de la enfermedad (Ficat-Arlet II o III), pero que se deben limitar a pacientes con ángulo necrótico de Kerboul (suma de ángulos que bordean el secuestro en anteroposterior y vistas de rayos $X$ laterales) inferiores a $200^{\circ}$. Históricamente, hace 
25-30 años, se pensaba que la variación, con o sin osteotomías de medicalización, era suficiente, pero más tarde, las osteotomías de flexión (Kempf) o rotacionales (Sugioka) comenzaron a ganar terreno. Un posible problema con las osteotomías es que pueden hacer que la conversión a artroplastia total de cadera sea más difícil técnicamente y menos exitosa a largo plazo.

- Artroplastia. La mayoría de los pacientes se dirigen a un cirujano ortopédico en etapas avanzadas de la enfermedad, después del colapso de la cabeza femoral. El reemplazo total de la articulación es el tratamiento de elección en las etapas finales de la necrosis avascular o cuando la articulación se destruye irreversiblemente. El procedimiento se puede realizar de muchas maneras, según las preferencias del cirujano. El cirujano puede usar varios enfoques (anterolateral, Hardinge, posterolateral, mínimamente invasivo, etc.) y puede emplear una gama aún más amplia de implantes. Las endoprótesis totales de cadera clásicas (cementadas o, preferiblemente, sin cemento) representan la mayoría de los implantes utilizados, pero los cirujanos y los pacientes recurren cada vez más a la artroplastia de revestimiento, un procedimiento que tiene varias ventajas. A diferencia de los implantes clásicos que emplean un par de fricción de metal en polietileno, cerámica o, más recientemente, Oxinium se coloca copas de polietileno, la artroplastia de revestimiento emplea un par de fricción metal sobre metal que es el más cercano a la articulación de la cadera normal. Mediante esta técnica, el acetábulo se reemplaza con una copa de metal sin cemento, muy similar a su predecesor clásico, pero en lugar de extraer toda la cabeza y el cuello femoral (como es el caso de los implantes clásicos), la cabeza femoral se prepara retirando su cartílago. y una capa delgada de hueso, preservando así la mayor parte del stock óseo. Se cementa un implante femoral de gran diámetro (generalmente de más de $44 \mathrm{~mm}$ ), lo que garantiza una estabilidad superior contra la dislocación. Además, el diseño de estos implantes garantiza un rango de movimiento aún mejor, que a veces puede ser crucial para pacientes jóvenes y activos. Además, el rejuvenecimiento de la artroplastia conserva más del stock óseo del paciente, una característica que se vuelve cada vez más importante, teniendo en cuenta la corta edad del paciente, lo que significa que probablemente necesitará una segunda y, a veces, incluso una tercera intervención algún tiempo después. La artroplastia total de cadera, ya sea clásica o repavimentada, produce excelentes resultados, con alivio inmediato y duradero del dolor y permite una movilización temprana y un retorno más rápido a un estilo de vida activo. Sin embargo, varios autores han observado que hay un fracaso anterior del reemplazo total de cadera en la osteonecrosis que en pacientes con otro diagnóstico de la misma edad. Esto probablemente se deba a la remodelación anormal de los huesos y al hundimiento de la prótesis debido a la mala calidad ósea del fémur proximal. Otros factores pueden incluir enfermedad sistémica en curso, defectos en el metabolismo mineral, uso de esteroides, alto nivel de actividad en pacientes jóvenes y aumento de peso corporal.

\section{Conclusión}

Con los años, a medida que nuestro conocimiento de esta enfermedad ha mejorado, se han perfeccionado varios métodos quirúrgicos y conservadores de tratamiento. Su objetivo es preservar la cabeza femoral y evitar su colapso durante el mayor tiempo posible, teniendo en cuenta al mismo tiempo que el tratamiento máximo y definitivo es el reemplazo total de cadera. Se deben hacer esfuerzos para retrasar el momento en

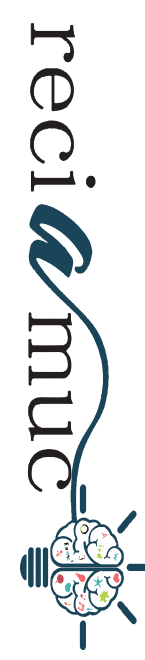


que se necesita una artroplastia, sin comprometer la posibilidad de un reemplazo directo de cadera.

Aunque se han realizado amplios estudios sobre la necrosis avascular de la cabeza femoral, todavía sabemos poco sobre su

\section{Bibliografía}

- $\rightarrow$ Aiello, M. (1 de August de 2008). Obtenido de Avascular Necrosis of the Femoral Head: http:/ /emedicine.medscape.com/article/ 386808

Aldridge, J., \& Urbaniak, J. (2004). Avascular necrosis of the femoral head: etiology, pathophysiology, classification, and current treatment guidelines. . Am J Orthop, 327-332.

Bolívar, J. (2015). Investigación Documental. México. Pax.

Castro, J. (2016). Técnicas Documentales. México. Limusa.

Davila, A. (2015). Concepto de terminos cientificos. Caracas: Oasis.

Jeanne, K., Tofferi, J., \& Gilliland, W. (24 de October de 2008). Avascular Necrosis. Obtenido de http:/ / emedicine.medscape.com/article/ 333364 patogénesis y, por lo tanto, sobre el mejor método para tratar esta enfermedad. El tratamiento médico futuro debe estar dirigido a la causa de esta enfermedad, lo que ralentiza o incluso detiene la evolución de la necrosis avascular y, por lo tanto, retrasa lo más posible la necesidad de cirugía.

Jeanne, K., Tofferi, J., \& Gilliland, W. (24 de October de 2008). Avascular Necrosis. Obtenido de http:/ / emedicine.medscape.com/article/ 333364

Marti-Carvajal, A., Dunlop, R., \& Agreda-Perez, L. (2004). Treatment for avascular necrosis of bone in people with sickle cell disease. Cochrane Database Syst Rev.

Marti-Carvajal, A., Dunlop, R., \& Agreda-Perez, L. (2004). Treatment for avascular necrosis of bone in people with sickle cell disease. Cochrane Database Syst Rev.

Sarikaya, I., Sarikaya, A., \& Holder, L. (s.f.). The role of single photon emission computed tomography in bone imaging. Semin Nucl Med , 3-16.

Schoenstadt, A. (05 de November de 2008). Avascular Necrosis. Obtenido de http://bones.emedtv. com/avascular-necrosis/avascular-necrosis.htm

\section{CITAR ESTE ARTICULO:}

Quinaluisa Erazo, C., Veintimilla Pesantez, J., Pérez Vásquez, M., \& Aldás Ibujes, K. (2020). Necrosis avascular de la cabeza femoral. RECIAMUC, 4(1), 4-14. doi:10.26820/reciamuc/4.(1).enero.2020.4-14 